\title{
Prevention and Control of Chagas Disease - An Overview
}

\author{
A. R. L. Teixeira ${ }^{1 *}$, C. Gomes ${ }^{2}$, A. C. Rosa ${ }^{1}$, P. F. Araujo ${ }^{1}$, C. E. Anunciação ${ }^{2}$, \\ E. Silveira-Lacerda ${ }^{2}$, A. B. Almeida ${ }^{1}$ and S. Petrofeza ${ }^{2}$ \\ ${ }^{1}$ Chagas Disease Multidisciplinary Research Laboratory, Faculty of Medicine, University of Brasilia, \\ Brazil. \\ ${ }^{2}$ Center for Research and Prevention of Neglected Diseases, Institute of Biology, Federal University of \\ Goiás, Brazil. \\ Authors' contributions \\ All authors had substantial contribution to the literature review, conception of the work, revised the \\ work critically for important intellectual content, approved the final version and agree to be \\ accountable for all aspects. \\ Article Information \\ DOI: 10.9734/ISRR/2018/42594 \\ (1) Anjana Verma, Assistant Professor, Department of Community Medicine, Geetanjali Medical College, Geetanjali Medcity, \\ India. \\ Reviewers: \\ (1) Alejandro Carabarín-Lima, Benemérita Universidad Autónoma de Puebla, México. \\ (2) Natalia Indira Vargas Cuentas, Universidad de Ciencias y Humanidades, Peru. \\ Complete Peer review History: http://www.sciencedomain.org/review-history/25651
}

Review Article

Received $10^{\text {th }}$ May 2018 Accepted $19^{\text {th }}$ July 2018 Published $24^{\text {th }}$ July 2018

\section{ABSTRACT}

Chagas disease is the main cause of heart failure and sudden death in the Western Hemisphere. The literature of the last decades reported on the changing epidemiological profiles of Chagas disease, which now threats the human population in the cities. The exodus of the Latin America people to the Northern Hemisphere explains the growing concern in countries where the transmission of Trypanosoma cruzi was accidental or transferred from a mother to her offspring. Herein, we present the evidence of the possible acquisition of the $T$. cruzi infection by sex. The staggering demonstration of the transmission of the T. cruzi infections from males and females to naïve mates by intercourse introduces substantial changes in the surveillance of the Chagas disease. Notably, the sexual transmission of the $T$. cruzi introduces changes in the concepts of medical care, prevention and control; specifically, the risk for the vertical transfer of the parasiteinduced kDNA mutations, underpinning the genetically driven autoimmunity, inheritance, and pathogenesis associated with multifaceted clinical manifestations of Chagas disease with high 
ratios of morbidity and mortality. In this regard, the endemics require much paradigm research with new approaches and innovation technologies, aiming at its control. For example, the recent knowledge anticipates useful measures for preventing the potential forthcoming pandemic Chagas parasites. A long-lasting multicenter research program is needed for creative, drug discovery for curtailment of Chagas disease. Meanwhile, the prevention shall rely on the education, information, and communication program for health.

Keywords: Trypanosoma cruzi; sexual transmission; pathogenesis; drug development; prevention; education.

\section{ACHRONYMS}

ATPase : adenyltriphosphatase.

Benznidazole and Nifurtimox : nitroderatives used for the treatment of Chagas disease.

$C C D$ : chronic Chagas disease.

CSB : kinetoplast minicircle constant sequence block.

CYP5128-30 : fenarimol herbicide azole inhibitor of T. cruzi.

$\mathrm{EICH}$ : education, information, communication for health.

elF2a : elongation factor $2 a$.

ELISA : enzyme-linked immunosorbent assay.

IFI : immunofluorescence indirect.

ik2 : eukaryote kinase subunit 2.

EMBL : European molecular biology laboratory.

ERV : endogenous retro virus.

LINE-1 : long interspersed nuclear element-1.

$M A L-R$ : long terminal repeats.

NAT : nucleic acid test.

nDNA : T, cruzi nuclear DNA.

kDNA : T. cruzi mitochondrion kinetoplast DNA;

PI3K : phosphate inositol 3 kinase.

$P C R$ : polymerase chain reaction.

${ }^{32}$ P-dATP : P'-(32)P-labeled; 2'-deoxyadenosine triphosphate.

\section{INTRODUCTION}

The Kinetoplastid flagellates in the Family Trypanosomatidae (from greek; trypanon, auger; soma, body) include Trypanosoma cruzi, the agent of the American trypanosomiasis [1,2]. The $T$. cruzi belongs to the Stercorarian group of flagellates that accomplishes its life cycle in the gut of the invertebrate host; the insect sucks in the blood trypomastigote, which transforms into epimastigote in the foregut and reverts to infective metacyclic trypomastigote in the hind gut. The requirement of an invertebrate host for completion of the protozoa lifecycle ascribes to the beginning of the $T$. cruzi enzootic infection of mammals that dwell in the American Continent [3-5]. The enzootics include reservoirs of the orders Marsupialia, Edentata, Chiroptera, Carnivora, Artiodactyla, Rodentia and Primates, upon whom triatomine bugs prey and transmit $T$. cruzi [5-7]. The spreading of the $T$. cruzi infections requires a broad diversity of triatomine vectors and mammalian hosts [5-10].
In past decades, the epidemiologic profiles of American trypanosomiasis and Chagas disease underwent drastic changes due to the rural exodus and, therefore, now they become an emerging urban problem [6-18]. The changes of the profiles follow pattern of the migration of $T$. cruzi-infected people from the South America continent and their settlement in the Northern Hemisphere [18].

Herein, we review the literature that describes the recent change of epidemiologic profiles of the Chagas disease. The review integrates the available data and information in men and in laboratory animals with growing concern about new technologies for preventing Chagas disease that threatens the people worldwide [7-19]. With this respect, this review article presents up-todate parasitological, clinic, cell biology, immunologic, genetic, pathology and the scanty epidemiologic information for the attention of the scientific community and for the health 
authorities. The aim of this review is to spurring a long-lasting program, aiming at a new drug to treat the carrier and to eradicate the endemic $T$. cruzi infections and control of the sexual transmission of the T. cruzi [18-20]. This review anticipates that the curtailment of Chagas disease needs international solidarity and multicenter research groups to undertake efficacious, creative, prevention paradigm achievements. In this regard, a mass-media Education, Information, and Communication for Health $(\mathrm{EICH})$ program to prevent Chagas disease in the forthcoming decades can no longer be neglected.

\section{TRANSMISSION ROUTES}

The main public health problem initiated, possibly, with the accidental triatomine bugtransmission of the $T$. cruzi infections to human [21-23]. However, the route for the bug hindgut's trypomastigote entry into the host's body and parasitic cell growth, is cumbersome: $i$ ) in the absence of systematic information, it is assumed that most acute $T$. cruzi infections stem from triatomine bug's bite, supposedly, in hinterland endemic areas; ii) incomplete knowledge about the bug's feces $T$. cruzi contamination ratios in countless ecotypes of the America continent [2430]; iii) triatomine bug's night habits, frequency of accomplishing a full meal and ratios of $T$. cruzi contamination, is mostly unknown [27-29]; iv) quick feeding ability to obtaining a full blood meal, distension of the abdomen and bug immediate defecation, remains to be determined [27-29]; v) triatomine bite site scratch-induced allergic reactions and vase dilation promotes bug's proboscis cannulating and suck-in a full blood meal, defecation and spread of the excreta, and the T. cruzi contaminates the human body [27-29]; nonetheless, in the absence of an allergic reaction and vase dilation the prey does not aware about the annoyance that incites the scratching, which renders much difficult a full completion of the time consuming blood meal, defecation and possible contamination of the host's body [24-31]; vi) the frequency of the hypersensitivity lesion at the port of entry of the parasite in the skin (Chagoma) or in the eye conjunctiva (Romaña's sign) is, possibly, less than circa 1 out of 1000 acute infection $[5,6]$.

The spectrum of drawbacks translates an array of difficulties towards collecting family information about bug's transmitted $T$. cruzi infections to the human population. However, the early recognition of the vector-transmitted $T$. cruzi infections posited first in the rank among routes of the $T$. cruzi contamination of humans $[18,23]$. In this regard, further investigation is needed to determine whether the ratios of $T$. cruzi-positive triatomine infestations correlate to the prevalence of Chagas disease in the cities.

The acquisition of the T. cruzi infections per os is the most ancient route for the $T$. cruzi contaminating the insectivorous mammals' reservoirs in the wilderness [31-33]. A few epidemiologic studies refer to the outbreaks of acute Chagas disease in humans, possibly, by imprudent ingestion of food contaminated with the triatomine bug's feces $[2,32,33]$. Next, the acquisition of the $T$. cruzi infections by blood transfusion is reported; lately this route of the infection acquisition is considered, carefully, as well in several countries of the Northern Hemisphere [34-36]. Occasionally, the T. cruzi infections are acquired by accident in the laboratory and by organ transplantation [36].

The transmission of the $T$. cruzi from the Chagas mother to her offspring is an important route of the infections in humans [36-38]. The congenital transmission of Chagas disease refers to the in uterus infection acquisition by the offspring. However, pregnancy depends upon partnership and male contribution to the in uterus $T$. cruzi infections awaits investigation. In the course of the pregnancy, the chagasic woman may undergo early embryo resorption, miscarriage, death birth, or neonatal death; live births can either be $T$. cruzi-infected or infection-free, healthy baby. Additionally, the infection-free, healthy progeny can inherit the $T$. cruzi mitochondrion minicircle kDNA sequences into the genome [7].

\section{CHAGAS DISEASE}

The routes of acquisition of the $T$. cruzi infections impose either the silent American trypanosomiasis or the clinically manifested Chagas disease. Fair accounts of the prevalence of those patients are missing and, therefore, the epidemiologic reports on morbidity and mortality due to the $T$. cruzi infections are rather inconclusive [6]. In the absence of disease manifestation, chronically infected humans are intermediate-phase carriers or reservoirs of the T. cruzi infections $[7,37,38]$. A majority of chronically infected individuals remains in the intermediate phase and their life expectancy is similar for non-infected individuals [7]. 
The field studies show that some chronically infected individuals develop chronic Chagas disease (CCD) usually over $\sim 30$ years after the acquisition of the $T$. cruzi: $94.5 \%$ are cases affected by heart trouble, among which $38.5 \%$ die suddenly, and $56 \%$ succumb to heart failure $[6,7]$. Sudden deaths are associated with arrhythmias and heart rate turbulence. In addition, megaesophagus and megacolon are revealed in $5.5 \%$ of chronically infected people. A gamut of neurologic syndromes and clinical dysfunctions are manifestations of Chagas disease, yet, scarcely studied [7-10]. Moreover, CCD clinic and pathologic features, which are indistinguishable from idiopathic inflammatory dilated cardiomyopathy, have the differential diagnosis set by the NAT-nDNA test $[6,7]$.

\section{DIAGNOSIS}

\subsection{Trypanosoma cruzi in the Blood}

The direct microscopic demonstration of $T$. cruzi in the blood is recorded in the early phase of the infection. Also, the parasite is detected by concentration methods, such as blood culture and xenodiagnoses (having a clean bug to feed upon the patient's blood). For the epidemiologic surveys, however, these diagnostics approaches are out of reach by a majority of the $T$. cruzi intermediate phase chronically infected population, in a lack of late clinical CCD manifestations.

\subsection{Immune Diagnosis}

In the absence of a direct demonstration of the $T$. cruzi in patient's blood the diagnosis relies on searches for the specific antibody. The acutely infected people are recognized by detecting IgM antibody class or IgG serum conversion usually after the second week of infection. Currently, highly sensitive, but relatively less specific indirect hemagglutination, enzyme-linked immunosorbent assay (ELISA), and indirect immunofluorescence (IFI) tests are routinely used [41-42]. However, none of these assays affords complete sensitivity and specificity, because the host's immune system sees a plethora of parasitic antigens and clonal selection of immune responses $[6,7,41,42]$. A word of caution is required because a grey zone of inconclusive assays that yield cross-reactive antibodies in the serum of patients with Leishmania sp., Mycobacterium leprae and $M$. tuberculosis, Treponema pallidum, or autoimmune diseases [7,42]. Largely, the employment of recombinant or synthetic peptide antigens diminishes the sensitivity of the assays, and these concerns bear practical consequences $[38,39]$. With this respect, caution is required in order to avoid an accidental $T$. cruzicontaminated blood transfusion. The health systems should no longer rely solely on testing the humoral immunity specific $T$. cruzi antibody [7,41-47]. Nonetheless, the electrocardiograph recordings in series of patients from endemic regions show cases with the positive serum anti$T$. cruzi lgG, increased heart size in the chest $X$ rays, and arrhythmias with features of chronic Chagas disease [48-50].

\subsection{Nucleic Acids Test (NAT)}

The accuracy of the diagnoses of the $T$. cruzi infections to the point-of-care for the assessment and curtailment of the epidemics, and as well for the health care delivery to thousands of people aggravated by the yet incurable Chagas disease, is fundamental $[43-47,51,52]$. Therefore, the diagnosis of the sexually transmitted infection requires the flagellate protozoa footprint [38-40, 43-47], because the specific IgG antibody is absent in the immune tolerant progeny of the $T$. cruzi-infected parents [38,51-54]. The immune tolerance showed in the chicken model system refractory to the $T$. cruzi infection after the $10^{\text {th }}$ day of incubation [38,47,51-54]. The chicks hatched from $T$. cruzi-inoculated eggs, which grow to adult life, do not raise specific antibody ( $Y$ immunoglobulin) after challenge with the formalin killed parasite $[53,54]$.

The NATs detect $T$. cruzi nuclear DNA (nDNA) and its kinetoplast DNA (kDNA) in somatic cells, and as well in germ cell line of the reproduction systems immune privilege organs [38,43-47,5154]. Moreover, the NAT-nDNA test is an indispensable condition for the diagnosis of $T$. cruzi infection in immune-tolerant patients, in the absence of the specific antibody. The development of throughput digital PCR platforms [47] needed to testing blood donor candidates, so as deferral of those NAT-nDNA positive blood donors, and to surveying accurate prevalence and correct information on the epidemiology of American trypanosomiasis and Chagas disease.

In so far, the NAT polymerase chain reaction (PCR) is the basis for research laboratories diagnosis of the $T$. cruzi infection and detection of congenital Chagas disease at birth [38,43-46]. Its employment ensures tidy laboratory practices to achieve a specific diagnosis of the highly 
repetitive sequences in the flagellate protozoa, showing homology with human chromosomes; and throughput platform required for large-scale epidemiologic surveys. A gamut of nDNA primer sets is available for in-house PCR diagnosis of the $T$. cruzi infections with variable sensitivity and specificity [38, 44,47]. The Tcz1/2 primers set that targets a 188-nt telomere DNA repeat in the $T$. cruzi genome [48] are used in the familybased studies [38-51]. The amplicons resolved in agarose gels and transfer to positively charged nylon membranes hybridize to the 188-nt $\alpha^{32} p$ dATP labeled probe, subsequently subjected to $X$-Rays film exposure during variable periods. The positive amplicons are subjected to cloning and sequencing. The bioinformatics analyses reveal the 188-nt nDNA footprint in the blood and in the semen samples. This in-house NAT is the point-of-care diagnosis of an active $T$. cruzi infection [38, 43-46]. Therefore, gold standard NATs are required to guarantee integrity, purity, and adequacy of blood bank supply donor deferral and pathogen inactivation of blood components. These efforts should be supported by the health care networks for prevention of an emerging $T$. cruzi threat; laboratory procedures quality control assessment by skilled personnel are needed to monitor all steps prior to the blood transfusion and for delivery of the health care. Additionally, the importance of the NAT tests for diagnosis of the $T$. cruzi infection and CCD cannot be underemphasized because the crosssection epidemiological surveys based on the IFI serological assessments of the specific antibody determine neither the accurate prevalence of the $T$. cruzi infections nor the ratios of the parasite sexual transmission in the human population [38, 41-46].

\section{TRANSFER OF KINETOPLAST DNA (kDNA) FROM AMERICAN TRYPANOSOMES TO HUMANS}

The studies showed transfer of the mitochondrial DNA (kDNA) minicircles sequences from $T$. cruzi to the rabbit's chromosome [56], and it was further showed that the Chagas rabbit progeny retained the protozoan minicircle sequence mutation into the genome [7, 52-56]. The chicken family studies spurred the human families' investigations on the transfer of the kDNA sequences from Chagas disease parents to their progeny [54-56]. The founders of study families showed kDNA minicircle sequences integrated into the Long Interspersed Nuclear Element-1 (LINE-1, equivalent to CR1 in the chicken) of the human genome. In addition, the
kDNA minicircle sequences integrated at various locations, such as structural, cell growth and differentiation, immune responses and at important biochemical pathways checkpoint genes [52-56].

The development of Chagas heart trouble in the chronically infected human population is an ominous sign because the cases succumb to the autoimmune inflammatory cardiomyopathy either suddenly or in the range of six months to two years after the beginning of clinical symptoms and failure, and, therefore, it is unusual to find those cases in a cross sectional field study. Nevertheless, the family study showed the accumulation of a minimum of 4 to $8 \mathrm{kDNA}$ mutations into the retrotransposable LINE-1 located at various chromosomes of CCD cases with clinically manifested heart insufficiency [7, $38,52-56]$.

The chagasic parental (F0) vertically transferred the $T$. cruzi kDNA minicircle sequences to the $F 1$ and $F 2$ progeny, which integrated into the genome [38]. Additionally, the families study population showed that somatic cells had the $T$. cruzi NAT-nDNA positive tests in family members and that sperm donors had the active $T$. cruzi infection. These findings suggest that intercourse is a sustainable route for transmission of the $T$. cruzi infection [38]. The clinic and epidemiologic importance of these findings address to the flagellate life-long infection, the overtime accumulation of the mitochondrion kDNA minicircle mutations and Chagas disease multifaceted manifestations, in which parasiteinduced mutations underpinning the genetically driven autoimmune pathogenesis, remarkably, features of host-parasite relationships and sprouting human disease.

\section{SEXUAL TRANSMISSION OF THE AMERICAN TRYPANOSOMES}

In the last decades, the growing number of Chagas disease in the Northern Hemisphere inhabitants ever exposed to the insect vectors suggests the possibility of acquisition of the $T$. cruzi infections by the intercourse $[7,38]$. In this regard, the $T$. cruzi infections encrypted in immune privilege body niches, in the absence of inflammatory infiltrates, make the grounds for the parasitic growth [57-63] in the reproductive organs (Fig. 1) and, thus expelling the protozoan in the semen (Fig. 2) and in the uterine secretions $[38,57]$. 


\subsection{Family Study}

The timely demonstration of the sexually transmitted $T$. cruzi in families' document the epidemic bursts of the infections in the population that received health care at the Center for Chagas Disease Surveillance. Among individuals in the families, there were cases of the acute $T$. cruzi infections showed by direct demonstration of the blood trypomastigotes. However, the epidemiologic history of the study population revealed the absence of the triatomine-bite transmission of the infections and, also, the other routes of the infection-acquisitions were denied.

In the family study, each acute case treated with the anti-trypanosome nitro derivative benznidazole ( $5 \mathrm{mg} / \mathrm{kg} /$ day) for 60 days, and all family members received health assistance for five years. The individuals in the families yielded blood samples at 1, 2, and 3 years set points for serum and DNA collection. Interestingly, the NAT-nDNA test was consistently positive in $76 \%$ independent assessments of blood samples collected at three different set points: among these, $28.4 \%$ tested IgG positive. This broad discrepancy explained by the immune tolerance, in the absence of the antibody in the majority of NAT-kDNA positive individuals, since the sexually transmitted $T$. cruzi could reach the early embryo before the development of the immune system [38,52-57]. Additionally, the NAT-kDNA test was positive in $92.6 \%$ family members (EMBL HG008116 to HG008708). The difference between the results obtained by the NAT-nDNA and the NAT-kDNA approaches addresses to the mutations resulting from the integration of the minicircle DNA sequences into the human genome in the absence of live T. cruzi infections. (Fig. 3).

In the absence of the T. cruzi antibody, the NAT analyses of the germ cells collected from subjects in the family study confirmed the experimental data $[38,52-56]$ showing that the $T$. cruzi infections acquired during early embryo growth do not bear the specific antibody, whereas those acquired during fetal development do not undergo immune-tolerance and, thus reveal the specific antibody. These analyses showed the $T$. cruzi nDNA- and kDNA-specific bands in the somatic cells of the blood and in the haploid semen samples examined [38, 57-63] (Fig. 4). The data that suggest the sexual transmission of the $T$. cruzi infections confirmed by other experiment, in which the injection of 100 $\mu l$ aliquot of NAT-nDNA positive Chagas patient's semen into the peritoneal cavity or its infusion into the vagina of mice documented the infectivity of the $T$. cruzi present in the human ejaculate: one month thereafter, the growing $T$. cruzi amastigote nests appeared in the heart and skeletal muscles, and in the lumen of the vas deferens and uterine tube (Fig. 4).

The early epidemiology based serological surveys conducted in the spare individual estimate that the prevalence of the $T$. cruzi infections averages $7 \pm 3$ percent of the Latin America population $[6,49]$. The family study NAT assessments revealed much high ratios of the $T$. cruzi infections in male and female family's founders and progeny mates [38,52-56]. However, the so-called congenital transmission of the infection means that the woman only bears for the vertical $T$. cruzi transmission to her baby. Certainly, such prejudice definition is in the lack of scientific understanding, because it omitted the inevitable roles played by both mates during the bi-partisan sexual reproduction [38]. With this respect, further family-based studies at various ecosystems are required to determine the role played by the mate's sexual intercourse in the transmission of the infectious agent retained in the immune privilege reproductive organs. The immune privilege is the absence of destructive inflammatory reactions to microbes' antigen loads in important body structures in the brain, eyes, and reproductive organs, accomplishing sensitive functions, reproduction, and the species survival $[64,65]$. The immune privilege sets free the $T$. cruzi growth in the organs of the reproduction, whereas the immune tolerance [64] prevents the immune system rejection of the parasite antigen, because it is recognized as a component of its own body since early embryo life.

\subsection{Mouse Model System}

The pathology searches documented a gamut of the $T$. cruzi amastigote nests in the mouse uterine endometrium, ovary theca cells, and testicles' Sertoli cells and in the lumen of the seminiferous tube, and in the epididymis, seminal vesicles, prostate, and ureter [57-63]. Actually, the inoculation of the T. cruzi from the hindgut of a triatomine bug incited the replicative flagellates' growth [57] in the recipient mouse ejaculates (Fig. 2). Furthermore, the T. cruzi infections were documented upon Chagas patient's semen inoculation or infusion in naïve mice [38]. 


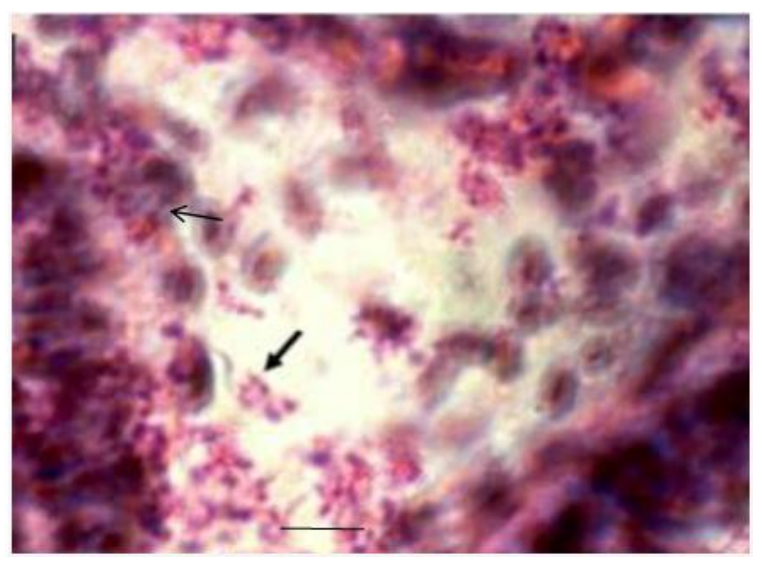

Fig. 1. The Trypanosoma cruzi infection in the seminiferous tube of a boy with the acute Chagas disease. Notice the round amastigote forms in the gonia blasts and clumps of amastigotes and trypomastigotes in the lumen of the tube (arrows). Bar, $10 \mu \mathrm{m}$. Microphotograph from Doctor Antonio Teixeira's file, 1970

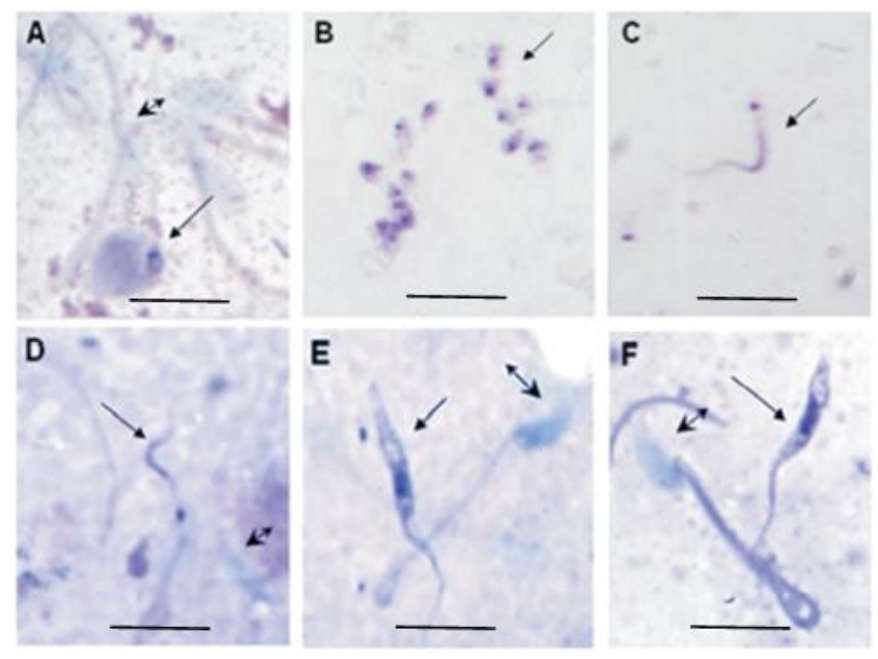

Fig. 2. The Trypanosoma cruzi parasitic forms in the semen ejaculate of acutely infected mice. The arrows indicate amastigote (A) within the head of a spermatozoan; free amastigotes (B) and trypomastigotes ( $C$ and $D$ ); and the epimastigote forms ( $E$ and $F$ ) in the semen ejaculates (arrows). Giemsa stained smears. Bars, $10 \mu \mathrm{m}$. Reprinted with permission from the Author and the Publisher [57]

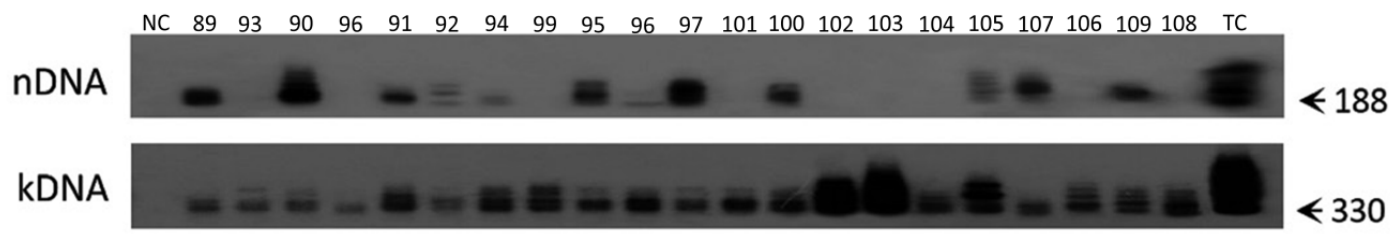

Fig. 3. The diagnosis of the Trypanosoma cruzi infections made by PCR and Southern hybridizations. Notice the nuclear DNA (nDNA) bands in 11 Chagas patients with the live infections, whereas 21 family members showed the mitochondrial DNA (kDNA) bands. Reprinted with the permission from the Authors and the Publisher [38] 

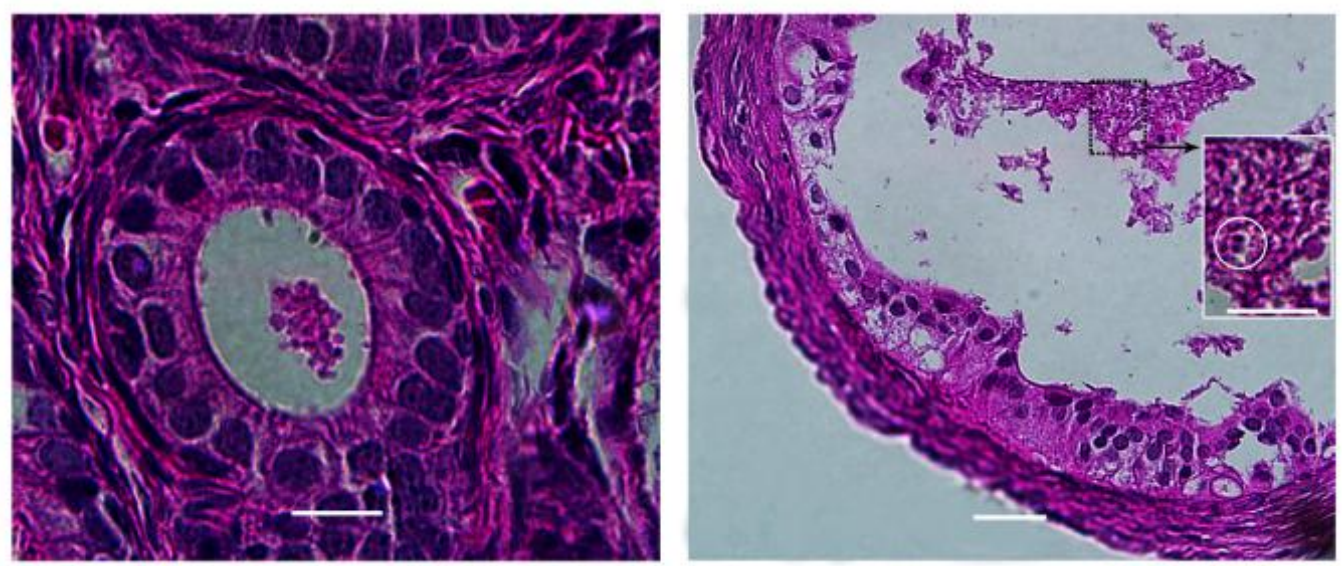

Fig. 4. The infectivity of the Trypanosoma cruzi from the human semen ejaculates upon aliquot instills into the peritoneal or into the vagina of naïve mice. Left) the $T$. cruzi amastigotes clump in the lumen of the vas deferens. Right) the $T$. cruzi amastigotes swarm epithelial cells of the uterine tube (insert, dividing amastigotes). Bars, $10 \mu \mathrm{m}$. Reprinted with the permission from the Authors and the Publisher [38]

Moreover, family studies about the $T$. cruziinfected male and female mice showed the transmission of the protozoa flagellates to naïve mates upon intercourse. In those experiments, each breeding pair placed in one cage inside a safe box avoided escaping. In experimental group-A, $10 \mathrm{~T}$. cruzi-infected males mated with 10 naive, control females. In experimental groupB, 10 T. cruzi-infected females mated with 10 naive, control males [38]. After breeding, groups $A$ and $B$ mates' yielded NAT-nDNA bands that is, the naive mates readily acquired the $T$. cruzi after the sexual encounter. The triplicate groups of independent experiments also showed that naive female or male mouse that sexually mated with a $T$. cruzi-infected male or female acquired the infection. Those nDNA-positive founders (F0) generated progeny $\mathrm{F} 1$ and $\mathrm{F} 2$ that they raised until six weeks of age. The founders' (F0) transmitted sexually the infections to $F 1$ progeny as shown by the nDNA-positive bands. The breeding of these nDNA-positive mates generated F2 progeny, which showed the nDNA bands that indicate the vertically acquired infections. In these experiments, $58.6 \%$ of the progeny had the NAT-positive nDNA assays, and $22 \%$ of the progeny had anti-T. cruzi antibodies. The results confirmed the absence of the specific antibody in a majority of F1 and F2 mice (78\%) that bore vertical $T$. cruzi infections by intercourse. After that, the tissue sections taken for microscopic examination showed the $T$. cruzi in the reproductive system of those mice [38]. In summary, those experiments suggest that few offspring $(22 \%)$ of chagasic mates had the $T$. cruzi-specific antibody, probably, because the infectious agent reached the fetus with a mature immune system.

\section{DRUG DEVELOPMENT}

The strategic for the treatment of Chagas disease should take into consideration that single infective T. cruzi initiates a lifelong infection [7]. Regardless of the route of the infection, after several cycles of division, the T. cruzi ends up in the reproduction organs immune privilege sites where it grows $[38,64]$. The amazing capability of the $T$. cruzi to thrive in the immune privilege mammalian reproductive system, so as to reach out the route to contaminate the host during intercourse; the species survival prevail transmission by sex and warrants the $T$. cruzi anthropozoonosis, and, therefore, this new concept shall guide the preventive medicine and public health measures for the control of American trypanosomiasis. This observation makes the absolute need of the parasite eradication in order to accomplish cure of Chagas disease and prevention of the sexually transmitted infections.

The lead drugs used for the treatment of the $T$. cruzi infections are nitro- derivative compounds nifurtimox [4-(5-nitro-phurylideneamino-) tetrahydro-4-4-1, 4-thiazine-1-1-dioxide], and benznidazole [N-benzyl-2-nitroimidazoleacetamide) [39-40, 49, 50, 81-84]. Both drugs achieve elimination of the $T$. cruzi infections in vitro but they fail to eradicate the live 
infections in experimental animals and in man $[38,40,66,67]$, possibly, because after-treatment non-phagocyte growing nest sustains the persisting parasite infection. The nitro-derivatives toxicity includes mutagenic, teratogenic, carcinogenic and sterilizing activities [68-76], which relies on the pteridine reductase activity [85-90] upon the lead and release of high-energy electron excited intermediates: ${ }^{-} \mathrm{NO}^{2},{ }^{-} \mathrm{HO},{ }^{1} \mathrm{O}^{2}$, $\mathrm{H}^{2} \mathrm{O}^{2}$ that bind to macromolecules and DNA. The drug toxicity targets the parasite and the mammalian host cell [7-79].

\section{NEW DRUGS FOR PREVENTING CHAGAS DISEASE}

The Chagas disease reported in five continents [25-37] has brought much attention from private and public organizations. In this new look, searches for safe, efficacious, low-cost drugs to curtailment of the epidemics needed. Consistently, the $T$. cruzi biochemical pathways checkpoints described herein suggest possible targets for drug development. The clinical trials with the 14a-demethylase (CYP51) that catalyzes the removal of the 14 a-methyl group from the scaffold revealed that posaconazole and ravuconazole inhibitors of sterol biosynthesis undergo treatment failure above that for benznidazole-treated patients [38,43-50]. The anti-trypanosome activity of lapachone-based 1,2,3-triazole naphthoquinones derivatives presented IC50/24 h 80.8 values at 6.8 and 8.2 $\mu \mathrm{M}$ activity which is above that of the anti- $T$. cruzi drug benznidazole. The nitro heterocycles fexinidazole prompted the discovery of the oxazole AN4169 (SCYX-6759) with anti-T. cruzi activity in a mouse model. Other leads derivatives of the herbicide fenarimol, which is an azole inhibitor of $T$. cruzi CYP5128-30. Meanwhile, the nitro derivatives benznidazole and nifurtimox registered for treatment of Chagas disease in Latin America [80-82]. In the countries of the Northern hemisphere the prescription of these drugs requires license from the health authorities.

Actually, the sustainable search for compounds from medicinal plants and drug development to kill the $T$. cruzi is needed, because most antibiotics derived from natural metabolites used for defense, reproduction, and survival [82-83]. The use of throughput biotechnologies for the systematic procurement and identification of active molecules in plants from various ecosystems, showing enormous biodiversity shall enhance the chance for obtaining a top lead drug without toxicity to eradicate the $T$. cruzi for prevention and treatment of Chagas disease. This is a milestone research endeavor.

\section{PREVENTING AMERICAN TRYPANOSOMIASIS AND CHAGAS DISEASE}

Over three decades the World Health Authorities were at odds with the devastating acquired immunodeficiency syndrome (AIDS). The epidemics caused the death of unaccountable people all over the world, in the lack of the specific diagnosis, except for the variety of opportunistic infections in the debilitating immune tolerant organism. Otherwise, International Health Authority Organizations consider Chagas disease minor epidemics that affect the poor people of Latin America, and the local Health Institutions claim that the disease is under control.

Meanwhile, the Chagas disease high morbidity and mortality ratios are growing burden upon the health systems worldwide and, therefore, it should not be neglected anyway (21-37). On the one hand, the Chagas disease unperceived epidemics [84-85] require the attention of the health systems because the potential of spreading the re-emerging transmission routes, including the sexually transmitted $T$. cruzi infections can no longer be underemphasized. On the other, the trophic network that enmeshes the $T$. cruzi in countless ecotypes in different ecosystems [4, 8], including the underground [86], embracing many species of insect vectors, most of which are not used to domiciliation are not susceptible to insecticides, and, therefore, the anthropozoonosis cannot be eliminated by the vector control strategy. To approach the magnificent problem the health personnel and scientists agree that time-consuming, unprecedentedly sustainable investigation programs are necessary, in order to create the knowledge new order to combat this threatening epidemic, urgently.

\subsection{Education Information and Communication for Health (EICH)}

The EICH program, aiming at the control of the transmission of the $T$. cruzi infections, is needed. The population is entitled to know about all routes of transmission of the $T$. cruzi infections: a) sexually transmitted; b) accidental contamination of health care workers and of research personnel, and through blood 
transfusion not deferred by the antibody assays; c) imprudent ingestion of contaminated food; d) insect-vector transmitted $T$. cruzi infections, which are limited to the American Continent. The a) and b) modes of transmission of the infections are prone to occur in the five continents. The basic EICH program to prevent the transmission of the silent $T$. cruzi infections shall take some experience of from AIDS mass-media guidelines, and the NAT nDNA exam performed early for the detection of the two weeks period in which the acute infections run in the absence of the specific antibodies $[38,87,88]$.

The EICH program should aim at the control of the epidemics through the involvement of the people in the community with the knowledge about the trophic networks that associate the insect vectors reservoirs with over one thousands mammal hosts in the indomitable nature [8]. The information about the several dozens of the insect species (triatomine bugs) transmitters of the $T$. cruzi, thriving in countless ecotypes, hiding away under tree's bark, in the mammals and in the birds' nests, as well under the rocks, and in burrows in the undergrounds, should be promptly delivered to the population [8, $11,17,18]$. They should know that a few triatomine bug species are susceptible to domiciliation, and those are main targets for elimination, to assure the family's protection at the household.

The EICH guidelines shall sustain the knowledge, saying that the education tempers the best health agent in the community. Additionally, to inform the people in the community about the genotoxicity of the nitroderivative pyrethroid pesticide sprayed in the house regardless of the inhabiting infants and adolescents $[68,69]$. In the case, the health authorities believe it is necessary to spray the pyrethroids an informed consent form with the Family Leader signature is a prerequisite for the insecticide spraying, in the household free of infants and adolescents.

A formidable effort is required for developing the $\mathrm{EICH}$ program aiming at the grass roots of the populations under potential exposures to the $T$. cruzi infections. Furthermore, the effective prevention measures shall sustain the concept that the $\mathrm{EICH}$ efficacy is a lot more protective of the family in the household than the spray of pesticides that do not reach most blood fasting triatomines dispersed in the hideaway environment. In addition, the citizen under the benefits of the EICH program shall fight to eliminate the nasty triatomine bug inside his house.

The citizens shall be informed about the constant increasing ratios of the $T$. cruzi infections in Latin America and worldwide. The continuous exodus of the people from Latin America to the Northern Hemisphere partly explains the many cases of the Chagas disease in the regions where the insect-vector does not exist. Meanwhile, the usefulness of the EICH program at school and at social organizations, with emphasis on the sociology, biological and health sciences, and mass-media communication, shall prevail worldwide in order to prevent the sexual transmission of the infections $[38,85]$. Moreover, unprecedented drug-discovery program needs for the parasite eradication and prevention of the sexually transmitted $T$. cruzi infections in the human population.

\section{DISCUSSION}

The trypanosome survival in invertebrate and in vertebrate animals include fine strategic checkpoints such as hiding away underground and association with countless ecotypes out of reach of skilled health personnel and insecticides. The plethora of countless factors, playing important roles in the multi-factorial chain of events related to transmission of the $T$. cruzi infections requires further studies in the laboratories and in the field. Meanwhile, the complexity of the $T$. cruzi life-cycle does not sustain the belief that currently used strategies are good enough for preventing the $T$. cruzi infections so as to safeguard the human population [8]. Such epistemological definition is essential because not yet defined cumbersome factors have been brought into play by an enormously complex chain of events and the $T$. cruzi encroaches in major ecosystems. The discovery of novel preventive and control strategies should be further investigated by scholars, before propagating the control of the $T$. cruzi infection and Chagas disease is achieved.

Actually, the sexually transmitted $T$. cruzi infection undergoes life-long encryption in the body, and, in the absence of clinic manifestations, the patients do not seek preventive medical care. In the absence of a drug to eradicate the $T$. cruzi infection, the $\mathrm{EICH}$ program is the corner stone for preventing the sexual transmission of the American trypanosomiasis and Chagas disease. 


\section{CONCLUDING REMARKS}

The $\mathrm{EICH}$ actions in the American Continent, concerning the prevention of the triatomines from close contact with the human population should be conducted directly in communities, elementary schools, churches and social clubs, and reinforced by social marketing and mass media communications. The population should be promptly informed about the modes of transmission of the $T$. cruzi infections that pose daily threats to human health. A realistic and affordable control of the sexually transmitted $T$. cruzi infections and the curtailment of Chagas disease rely on a robust $\mathrm{EICH}$ program.

The EICH preventive actions to halt the American trypanosomyasis and Chagas disease spread worldwide should be on the time table because the sexual transmissions of the T. cruzi infection run simultaneously with the transfer of the parasitic kDNA to the human genome. This frailty introduces a significant change in the concept of public health, and preventive medicine $[7,38,67]$. The silent parasite-induced, genetically driven autoimmunity [38,64-66,67] associates ubiquitous clinical manifestations with high ratios of morbidity and mortality several decades after the infection acquisition, and, therefore, a conjunction of measures is necessary to control the Chagas disease and its potential to become pandemic:

1. Chagas disease is a global problem that requires international solidarity, consortia, and exchanges of expertise in order to provide the means for protection of the population under risks of acquisition of the $T$. cruzi infections.

2. Education, Information, and Communication mass-media program $(\mathrm{EICH})$ undertaken, soon is the best, in order to obtain full compliance from the people's community, similar to that employed to control HIV-AIDS epidemics.

3. Safe new drugs needed to eradicate the cryptic $T$. cruzi infections: $i)$ to treat all the $T$. cruzi-infected people, so as to prevent the sexual and the accidental modes of transmission of the infections. ii) To arrest the cardiomyopathy: pre-empt the severe Chagas heart disease case, before the killing of sick bone marrow progenitors of the effector immune lymphocytes before healthy bone marrow transplantation $[64,65]$.

4. Development of throughput digital PCR platforms for assessing the population under risk of acquisition of the $T$. cruzi infections, so as deferral of NAT-nDNA positive blood donors.

5. The health personnel should share responsibility with the community leaders about the target-directed limited spraying of the pyrethroid insecticide in the triatomine bugs infested households.

\section{ACKNOWLEDGEMENTS}

This work was funded by the USA NIH grant R03 1164, and by the Brazilian Government PRONEX/FAPDF/ MCT/ CNPq/CAPES grant 193.000.589/2009. The funders had no role in study design, data collection and interpretation or decision to submit the work for publication.

\section{CONSENT}

It is not applicable.

\section{ETHICAL APPROVAL}

It is not applicable.

\section{COMPETING INTERESTS}

The authors have declared no conflict of interest exists.

\section{REFERENCES}

1. Lake JA, de la Cruz F, Ferreira PC, Morel C, Simpson L. Evolution of parasitism: kinetoplastid protozoan history reconstructed from mitochondrial rRNA gene sequences. Proc Natl. Acd. Sci. USA. 1988;85:4779-83.

2. Simpson AG, Lukes J, Roger AW. The evolutionary history of kinetoplastids and their kinetoplasts. Mol Biol Evol. 2002; 19:2071-6.

3. Lalonde RG, Holbein BE. Role of iron in Trypanosoma cruzi infection of mice. J Clin Invest. 1984;470:470-6.

4. Taylor MC, Kelly JM. Iron metabolism in trypanosomatids, and its crucial role in infection. Parasitol. 2010;137:899-917.

5. Coura JR. The main scenaries of Chagas disease transmission. The vectors, blood and oral transmissions. Mem. Inst. Oswaldo Cruz. 2015;110:277-82.

6. Teixeira AR, Nascimento R, Sturm NR. Evolution and pathology in Chagas 
Disease. Mem Inst Oswaldo Cruz. 2006; 101:463-91.

7. Teixeira AR, Hecht MM, Guimaro MC, Sousa AO, Nitz N. Pathogenesis of Chagas disease: Parasite Persistence and Autoimmunity. Clin Microbiol Rev. 2011; 24:502-630.

8. Teixeira AR, Monteiro SP, Rebelo JM, Argañaraz ER, Vieira D, Lauria-Pires, et al. Emerging Chagas disease: Trophic network and cycle of transmission of Trypanosoma cruzi from palm trees in the Amazon. Emerg Infect Dis. 2001;7:100 DOI: 10.3201/eid0701.700100

9. Chagas C. New human trypanosomiasis. Morphology and lifecycle of Schizotrypanum cruzi, the cause of a new human disease. Mem Inst Oswaldo Cruz. 1909;1:59-218.

10. Chagas C. A new human disease. Summary of etiological and clinical studies. Mem Inst Oswaldo Cruz. 1911; 3:219-275.

11. Reisenman CE, Lawrence $G$, Guerenstein PG, Gregory T, Dotson E, Hildebrand JG. Infection of Kissing Bugs with Trypanosoma cruzi, Tucson, Arizona, USA. Emerg Infect Dis. 2010;16:400-5.

12. Klotz JH, Dorn PL, Logan JL, Stevens L, Pinnas JL, Schmidt JO, et al. Kissing bugs: Potential disease vectors and cause of anaphylaxis. Clin Infect Dis. 2010;50:162934.

13. Kjos SA, Dorn PL, Yabsley MJ, Kitron U, Snowden KF, Logan KS, et al. Identification of bloodmeal surces and Trypanosoma cruzi infection in Triatomine Bugs (Hemiptera: Reduviidae) from residential settings in Texas, the United States. J Med Entomol. 2013;50:11261139.

14. Bussacos AC, Nakayasu ES, Hecht MM, Assumpção TC, Parente JA, Soares CM, et al. Redundancy of proteins in the salivary glands of Panstrongylus megistus secures prolonged procurement for blood meals. J Proteomics. 2011;74:1693-700.

15. Moffitt JE, Venarske D, Goddard J, Yates $A B$, de Shazo RD. Allergic reactions to Triatoma bites. Ann Allergy Asthma Immunol. 2003;91:122-8.

16. Hecht MM, Bussacos AC, Lozzi SP, Santana JM, Teixeira AR. Triatoma infestans chooses to feed upon immune prey. Am J Trop Med Hyg. 2006;75:893900.

17. Martínez-lbarra JA, Valencia-Navarro I, León-Saucedo S, Ibáñez-Cervantes G,
Bustos-Saldaña $R$, et al. Distribution and infection of triatomines (Hemiptera: Reduviidae) by Trypanosoma cruzi in the state of Michoacán, Mexico. Mem Inst Oswaldo Cruz. 2011;106:445-50.

18. Schofield CJ. Trypanosoma cruzi - the vector-parasite paradox. Mem Inst Oswaldo Cruz. 2000;95:95:535-44.

19. Yoshida N. Molecular mechanisms of Trypanosoma cruzi infection by oral route. Mem Inst Oswaldo Cruz. 2009;104 Suppl 1:101-7.

20. Shikanai-Yasuda MA, Carvalho NB. Oral transmission of chagas disease. Clin Infect Dis. 2012;54:845-52.

21. Blumental S, Lambermont M, Heijmans C, Rodenbach M-P, El Kenz H, Sondag D, et al. First documented transmission of Trypanosoma cruzi infection through blood transfusion in a child with sickle-cell disease in Belgium. PLoS Negl Trop Dis. 2015;9:e0003986

DOI: 10.1371/journal.pntd.0003986

22. Ries J, Komarek A, Gottschalk J, Brand $B$, Amsler $L$, Jutzi $M$, et al. A case of possible chagas transmission by blood transfusion in Switzerland. Transfuse. Med. Hemother. 2016;43:415-417.

23. Dias JCP, Amato Neto V. Prevention concerning the different alternative routes for transmission of Trypanosoma cruzi in Brazil. Rev Soc Bras Med Trop. 2011;44: suppl 2:68-72.

24. Cevallos AM, Hernández R. Chagas' disease: Pregnancy and Congenital Transmission. 2014;ID 40186.

25. Jackson $Y$. Congenital transmission of Chagas disease in Latin America. Emerg Infect Dis. 2009;15:601-3.

26. Bern C, Kjos S, Yabsley M. Montgomery. Trypanosoma cruzi and Chagas disease in the United States. Clin Microbiol Rev. 2011;24:655-681.

27. Klein N, Hurwitz I, Durvasula R. Globalization of chagas disease: A Growing Concern in Nonendemic Countries. Epidemo Res Int. 2012;ID 136793-806.

28. Liu Q, Zhou X-N. preventing the transmission of american trypanosomiasis and its spread into non-endemic countries. Infect Dis Poverty. 2015;4:60-71.

29. Jackson $Y$, Pinto $A$, Pett $S$. Chagas disease in Australia and New Zealand: risks and needs for public health interventions. Trop Med Int Health. 2014; 19:212-8. 
30. Gascona J, Bernb C, Pinazoa M-J. Chagas disease in Spain, the United States and other non-endemic countries. Acta Tropica. 2010;115:22-27.

31. Bartoloni A, Zammarchi L. Chagas disease in non-endemic countries, still a neglected public health issue. Infectious Diseases Unit, Department of Experimental \& Clinical Medicine; 2014. Florence, Italy.

Available:http://www.peah.it/2014/02/

32. Pérez-Molina JA, Norman F, López-Vélez $R$. Chagas disease in non-endemic countries: epidemiology, clinical presentation and treatment. Curr Infect Dis Rep. 2012;14:263-74.

33. Dorn PL, Perniciaro L, Yabsley MJ, Roellig DM, Balsamo G, Diaz J, et al. Autochthonous Transmission of Trypanosoma cruzi, Louisiana. Emerging Infect Dis. 2007;13:605-7.

34. Takeshi N, Miura S. Current situation of chagas disease in non-endemic countries. Junt Med J. 2015;61:389-95.

35. Albajar-Viñas $P$ \& Jannin J. The hidden Chagas disease burden in Europe. Euro Surveill. 2011;16:pii-19975.

36. Schmunis GA, Yadon ZE. Chagas disease: a Latin American health problem becoming a world health problem. Acta Trop. 2010; 115:14-21.

37. Castro AM, Vinaud M, Teixeira AR. Emerging chagas disease. In: Chapter 1. Chagas disease. A global Health Problem. Bentham Science Publishers, New York; 2009.

38. Araujo PF, Almeida AB, Pimentel FC, Rios A, Sousa A, Valente SA, Valente VC, et al. Sexual transmission of american trypanosomes in humans. New potential to become pandemic. Mem Inst Oswaldo Cruz. 2017;112:1-10.

39. Tarleton RL, Gürtler RE, Urbina JA, Ramsey J, Viotti R. Chagas Disease and the London Declaration on Neglected Tropical Diseases. PLoS Negl Trop Dis. 2014;8:e3219.

DOI:10.1371/journal.pntd.0003219 PMCID PMC4191937.

40. Braga MS, Lauria-Pires L, Nitz N, Rosa $A C$, Argañaraz ER. The treatment of Chagas disease patients with nitro derivative is unsatisfactory. Rev Inst Med Trop São Paulo. 2001;43:175-181. DOI:org/10.1590/S003646652001000300013

41. Teixeira AR, Vexenat A. The truthful meaning of immunologic exams used in the diagnosis of endemic diseases. Rev Soc Bras Med Trop. 1996;29:379-82.

42. Vexenat AC, Santana JM, Teixeira, ARL. Cross-reactivity of antibodies in human infections by the kinetoplastid protozoa Trypanosoma cruzi, Leishmania chagasi and Leishmania (Viannia) braziliensis. Rev Inst Med Trop São Paulo. 1996;38:177-85.

43. Britto CC. Usefulness of pcr-based assays to assess drug efficacy in chagas disease chemotherapy: value and limitations. Mem Inst Oswaldo Cruz. 2009;104(suppl 1):12235.

44. Hsieh $H$, Patterson AS, Ferguson BS, Plaxco KW, Soh HT. Rapid, Sensitive, and quantitative detection of pathogens DNA at the point-of-care via microfluidic electrochemical quantitative loop-mediated isothermal amplification (MEQ-LAMP). Angew Chem Int Ed Engl. 2012;51:4896900 DOI: 101002/anie.201109115

45. Virreira M, Truyens C, Alonso-Vega C, Brutus L, Jijena J, Torrico F. Comparison of Trypanosoma cruzi lineages and levels of parasitic DNA in infected mothers and their newborns. Am J Trop Med Hyg. 2007; 77:102-106.

PMID: 17620638

46. Mendes DG, Lauria-Pires L, Nitz N, Lozzi SP, Nascimento RJ, Monteiro PS, et al. Exposure to mixed asymptomatic infections with Trypanosoma cruzi, Leishmania brazilieinsis and Leishmania chagasi in the human population of the greater Amazon. Trop Med Int Health. 2007;12:629-36.

47. Troger V, Nieman K, Gartig C, KulhImeir D. Isothermal amplification and quantitation of nucleic acids and its use in mycrosystems. Nonomed. Nanotechnol. 2015;6:282-3.

48. Teixeira AR, Nitz N, Guimaro MC, Gomes C, Santos-Buch CA. Chagas disease. Postgrad Med J. 2006;82:788-95.

49. World Health Organization. Control: second report of a WHO Expert Committe. World Health Organ Tech Rep Ser. 2002; 905:1-109.

50. Balouz V, Aguero F, Buscaglia CA. Chagas disease diagnostic applications: present knowledge and future steps. Adv Parasitol. 2017;97:1-45.

51. Moser DR, Kirchhoff LV, Sonwlaon JE. Detection of Trypanosoma cruzi by DNA amplification using the polymerase chain reaction. J Clin Microbiol. 1989;27:147782. 
52. Teixeira AR, Gomes C, Nitz N, Sousa AO, Alves RM, Guimaro MC, et al. Trypanosoma cruzi in the Chicken Model: Chagas-Like Heart Disease in the Absence of Parasitism. PLoS Negl Trop Dis. 2011; 5:e1000.

53. Guimaro MC, Alves RM, Rose E, Sousa $A O$, Rosa AC, Hecht MM, et al. Inhibition of autoimmune chagas-like heart disease by bone marrow transplantation. PLoS Negl Trop Dis. 2014;8:e3384.

54. Nitz N, Gomes C, Rosa AC, D'Souza-Ault MR, Moreno F, Lauria-Pires L, et al. Heritable integration of kDNA Minicircle Sequences from Trypanosoma cruzi into the Avian Genome: Insights into Human Chagas Disease. Cell. 2004;118:175-186.

55. Hecht MM, Nitz N, Araujo PF, Sousa AO. Rosa AC, Gomes DA, et al. Inheritance of DNA transferred from American trypanosomes to human hosts. PLoS One. 2010;12:e9181.

56. Hecht MM, Nitz N, Araujo PF, Teixeira AR. Pathogenesis of Chagas disease. Lateral DNA transfer, genotype alterations and autoimmunity. Rev Soc Bras Med Trop. 2009;4(Supl 1):68-72.

57. Alarcón M, Moreno E, Colasante C, Yarbuh AL, Cáceres $\mathrm{K}$, Araujo $\mathrm{S}$. Presencia de epimastigotes de Trypanosoma cruzi en el plasma seminal de ratones con infección aguda. Bol Mal Salud Amb. 2011;51:237.

58. Lenzi HL, Castelo-Branco MT, PelajoMachado M, Oliveira DN, Gattass CR. Trypanosoma cruzi: compromise of reproductive system in acute murine infection. Acta Trop. 1998;71:117.

59. Carvalho LO, Abreu-Silva AL, Hardoim D de J, Tedesco RC, Mendes VG, Gonçalves da Costa SC et al. Trypanosoma cruzi and myoid cells from seminiferous tubules: Interaction and relation with fibrous components of extra cellular matrix in experimental Chagas disease. Int J Exp Path. 2009;90:52.

60. A Rios, M Ribeiro, A Sousa, F Pimentel, L Hagström, R Andrade, et al. Can sexual transmission support the enzootic cycle of Trypanosoma cruzi? Mem Inst Oswaldo Cruz, Rio de Janeiro. 2018;113(1):3-8.

61. Ribeiro M, Nitz N, Santana C, Moraes A, Hagström L, Andrade R, et al. Sexual transmission of Trypanosoma cruzi in murine model. Exp Parasitol. 2016;162: 1-6.

DOI: 10.1016/j.exppara.2015.12.017.
62. Martin DL, Lowe KR, McNeill T, Thiele EA, Roellig DM, Zajdowicz J, et al. Potential sexual transmission of Trypanosoma cruzi in mice. Acta Trop. 2015;149:15-8.

DOI: 10.1016/j.actatropica.2015.05.002.

63. Freitas JM, Pinto LA, Pimenta JR, Rodrigues LB, Gonçalves VF Teixeira SM. Ancestral Genomes, Sex, and the Population Structure of Trypanosoma cruzi PLoS Path; 2006.

Available:https://doi.org/10.1371/journal.pp at.0020024

64. Fujisaki J, Wu J, Carlson AL, Silberstein L, Putheti $\mathrm{P}$, Larocca $\mathrm{R}$, et al. In vivo imaging of Treg cells providing immune privilege to the haematopoietic stem-cell niche. Nature. 2011;474:216-219.

65. Schwartz RH. Historical overview of immunological tolerance. Cold Spring Harb Perspect Biol. 2012;4:a006908.

66. Lauria-Pires L, Braga MS, Vexenat AC, Nitz N, Simoões-Barbosa A, Tinoco DL, et al. Progressive chronic Chagas heart disease ten years after treatment with antiTrypanosoma nitro derivatives. Am J Trop Med Hyg. 2000;63:111-118.

PMID: 11388500.

67. Morillo CA, Marin-Neto JA, Avezum A, Sosa-Estani S, Rassi-Jr A. Randomized Trial of Benznidazole for Chronic Chagas' Cardiomyopathy. N Engl J Med. 2015; 373:1295-1306.

DOI: 10.1056/NEJMoa15075742015.

68. Teixeira AR, Silva R, Rizzo LV, Cunha neto $E$. Trypanocidal nitroarene treatment of experimental Trypanosoma cruzi infection does not prevent progression of chronic-phase heart lesions in rabbits. J Infect Dis. 1990;162:1420.

69. Teixeira AR, Cordoba JC, Souto Maior I, Solorzano E. Chagas disease: Lymphoma growth in rabbits treated with benznidazole. Am J Trop Med Hyg. 1990; 43:146-158.

70. Teixeira AR, Jabur E, Cordoba JC, Mayor ICS, Solorzano E. Alteração da resposta imune mediada por células durante 0 tratamento com Benzonidazol. Rev Soc Bra Med Trop. 1983;16:11-22.

71. Teixeira AR, Silva R, Cunha Neto E, Rizzo LV, Santana JM. Malignant, non-hodgkin's lymphomas in Trypanosoma cruzi-infected rabbits treated with nitroarenes. J Comp Pathol. 1990;103:37-48.

72. Teixeira A, Calixto M, Teixeira M. Chagas' disease: Carcinogenic activity of the 
antitrypanosomal nitroarenes in mice. Mutat Res. 1994;305:189-96.

73. Teixeira AR, Resende Filho J, Figueiredo FC. A clinical, immunological and pathological study in rabbits. Am J Trop Med Hyg. 1983;32:258-272.

74. Moreno SN, Docampo R. Mechanism of toxicity of nitro compounds used in the chemotherapy of trichomoniasis. Environ Health Perspect. 1985;64:199-208.

PMID: 3830698.

75. Miller R, Buttigan BE. Role of oxidants in microbial pathophysiology. Clin Microbiol Rev. 1997;10(1):1-18.

76. Traub-Cseko YM, Ramalho-Ortigão JM, Dantas AP, de Castro SL, Barbosa HS, Downing $\mathrm{KH}$. Dinitroaniline herbicides against protozoan parasites. The case of Trypanosoma cruzi. Trends Parasitol. 2001;17(3):136-141.

77. Dickinson BC, Chang CJ. Chemistry and biology of reactive oxygen species in signaling or stress responses. Nature Chem Biol. 2011;7:504-511.

DOI: 10.1038/nchembio.607.

78. Vermes A, Guchelaar HJ, Dankert DJ. Flucitosine: A reviews of its pharmacology, clinical indications, pharmaco-kinetics, toxicity and drug interactions. J Antimicrob Chemoth. 2000;46:171-179. DOI: 10.1093/jac/46.2.171.

79. Augusto LS, Moretti NS, TCP Ramos, Jesus TCL, Zhang M, Castilho BA, et al. A membrane-bound elF2 Alpha Kinase Located in Endosomes Is Regulated by Heme and Controls Differentiation and ROS Levels in Trypanosoma cruzi. PLoS Pathol. 2015; 11:e1004618.
DOI: 10.1371/journal.ppat.1004618.

80. Buckner SB, Navabi N. Advances in Chagas disease drug development. Curr Opin Infect Dis. 2010;23:609-616.

81. Chatelain E. Chagas disease drug discovery. Toward a New Era. J Biomol Screen. 2015;20:22-35.

82. Weniger B. Robledo S, Arango GJ, Deharo E, Aragón R. Antiprotozoal activities of Colombian plants. J Ethnopharmacol. 2001;78:193-200.

83. Bern C, Kjos S, Yabsley MJ. Trypanosoma cruzi and chagas disease in the United States. Clin Microbiol Rev. 2011;24:655681.

84. 84. Abad-Franck F, Gurgel-Gonçalves R, Gurtle RE. On bugs and bias: Improving Chagas disease control assessment. Mem Inst Oswaldo Cruz. 2014;109:125-130.

85. Hotez PJ, Dumonteil E, Woc-Colburn L, Serpa JA, Bezek S. Chagas disease: The new HIVIAIDS of the Americas. Plos Negl Trop Dis. 2012;6:e1498.

86. Lövy M, Šklíba J, Hrouzková E, Dvořáková V, Nevo E. Habitat and burrow system characteristics of the blind mole rat Spalax galili in an area of supposed sympatric speciation. PLoS One. 2015;10:e0133157.

87. Lloyd LS. Environmental Health Project Activity Report $n^{\circ} 56$. An assessment of health education materials for control of dengue and malaria in Honduras. USAID, Arlington, Virginia Environmental Health; 1999.

88. Williams-Blangero $\mathrm{S}$, VandeBerg J, Teixeira AR. Attitudes towards Chagas disease: Working to overcome the global impact of neglected tropical diseases. Cad Saude Publ. 1999;15:7-13.

(c) 2018 Teixeira et al.; This is an Open Access article distributed under the terms of the Creative Commons Attribution License (http://creativecommons.org/licenses/by/4.0), which permits unrestricted use, distribution, and reproduction in any medium, provided the original work is properly cited. http://www.sciencedomain.org/review-history/25651 